\title{
O compartilhamento de informações no transporte público com as tecnologias RFID e NFC: uma proposta de aplicação
}

\author{
Sharing information on public transport with RFID and NFC technologies: \\ an application proposal
}

Victor Nassar, Milton Luiz Horn Vieira

Universidade Federal de Santa Catarina (UFSC), Florianópolis, SC, Brasil

\section{Resumo}

O transporte público nos grandes centros urbanos brasileiros apresenta dificuldades relacionadas à informação disponibilizada aos usuários, tais como dados sobre linhas de ônibus, seus trajetos, terminais e horários, atualizações sobre as ocorrências de atrasos, entre outros. A aplicação de tecnologias possibilita compartilhar informações a fim de auxiliar o transporte urbano em geral. Nesse cenário, este artigo possui o objetivo de discutir a proposta de aplicação do Smart Bus, que utiliza as tecnologias NFC (Near Field Communication) e RFID (Radio Frequency Identification) para efetuar o compartilhamento de informações no transporte público. Após o referencial teórico, há um levantamento de projetos que utilizam as tecnologias NFC e RFID no transporte urbano e de aplicativos que contribuem com a gestão de informações sobre o trânsito. Em seguida, discutem-se o Smart Bus e as influências resultantes do compartilhamento de informação nos sistemas de transporte público. Por fim, verificou-se a contribuição da aplicação para o mapeamento do perfil das vias, ônibus e passageiros, localizando atrasos e provendo dados para a gestão do transporte público. Ressaltam-se ainda as implicações em relação à privacidade e à segurança dos dados de usuários, além da infraestrutura tecnológica necessária à implantação de projetos envolvidos nos conceitos das Cidades Inteligentes e da Internet das Coisas.

Palavras-chave: Transporte público. Tecnologias de informação. RFID. NFC. Cidades inteligentes.

\section{Abstract}

Public transport in large Brazilian cities present deficiencies related to providing timely information to users on data regarding route, terminals and schedules of bus lines, updates on cases of delay, among others. Technologies can be useful for sharing information to assist urban transport in general. In this context, the present paper has the objective to discuss the proposed application Smart Bus, which uses NFC (Near Field Communication) and RFID (Radio Frequency Identification) technologies to share information on public transport. With this theoretical framework, a survey of projects using NFC and RFID technologies in urban transport and applications that contribute to traffic information management was carried out. Then, the use of the Smart Bus application and the results of sharing information in public transport systems were discussed. At the end, the contribution of the application to mapping the profile of roads, buses and passengers, locating delays and providing data for

VN é designer, M. Sc, e-mail: victornassar@gmail.com MLHV é engenheiro, Dr., e-mail: milton.vieira@ufsc.br 
management of public transport was evident. Implications to privacy and security of passenger information, as well as to the technological infrastructure necessary for implementing projects under the concepts of Smart Cities and the Internet of Things, were notable.

Keywords: Public transport. Information technology. RFID. NFC. Smart cities.

\section{Introdução}

0 crescimento das grandes metrópoles é impulsionado por um desenvolvimento econômico e social que propicia benefícios - como oportunidades financeiras em educação, entretenimento, cultura, entre outros - e, ao mesmo tempo, uma série de problemas aos cidadãos das grandes cidades - como dificuldades relacionadas ao trânsito, poluição, segurança e saúde.

No cenário do transporte urbano brasileiro, sobretudo o chamado transporte público, as cidades ainda apresentam pontos cruciais para a melhoria da qualidade, como a infraestrutura das vias, as condições dos veículos, a acessibilidade a deficientes, a segurança interna e nos pontos de embarque e desembarque, os congestionamentos no trânsito etc. (Araújo et al., 2011). Em específico no que se refere à informação disponibilizada aos usuários do transporte público, observa-se que as dificuldades são referentes à divulgação precisa e em tempo real de dados sobre as frotas, os percursos e os horários dos ônibus, os períodos e os locais que apresentam maior fluxo de passageiros, as linhas que atuam em determinados pontos e terminais de ônibus, os atrasos, os congestionamentos etc.

São nessas problemáticas do desenvolvimento urbano que atuam os conceitos das Cidades Inteligentes (Smart Cities) e o da Internet das Coisas (IoT - Internet of Things), visando incorporar a adoção das tecnologias de informação e a comunicação ao contexto das situações cotidianas dos indivíduos. Busca-se explorar o suporte tecnológico a fim de gerar transformações positivas na sociedade, modernizando sistemas, gerenciando informações e automatizando processos. Dessa forma, a tecnologia consolida-se como importante aliada do ser humano para a realização de tarefas diárias, a partir da integração entre variados dispositivos, sensores e redes de conectividade (Atzori et al., 2010).

Entre as tecnologias atuantes nos conceitos de Cidades Inteligentes e de Internet das Coisas, incluem-se o NFC (Near Field Communication) e o RFID (Radio Frequency Identification), que, integrados a aparelhos eletrônicos, podem influenciar a experiência dos usuários em variadas soluções no transporte urbano, como a possibilidade da realização de compra de bilhetes eletrônicos, o acesso a catracas nos veículos, a obtenção e a transmissão de conteúdo (Ok et al., 2011).

Nesse contexto, este artigo traz uma abordagem sobre como as tecnologias de informação podem auxiliar sistemas de transporte urbano. Para a delimitação da discussão, propõe-se a aplicação do Smart Bus com o intuito de integrar em um único projeto as tecnologias RFID e NFC, atuando no compartilhamento de informações sobre o transporte público. Pode-se inferir por Gil (2009) que este trabalho caracteriza-se como descritivo, pois há a intenção de descrever particularidades de determinados eventos, analisando os dados com o intuito de estabelecer relações.

Para tanto, inicia-se com a construção do referencial teórico sobre a relação entre Cidades Inteligentes e Internet das Coisas, além das definições das tecnologias NFC e RFID, a fim de fundamentar a discussão sobre a relação entre tecnologias e transporte urbano. Em seguida, tem-se um levantamento de aplicações que utilizam as tecnologias NFC e RFID em sistemas de transporte de diferentes cidades no mundo. Segue-se com uma análise de aplicativos móveis que são direcionados a contribuir com a gestão de informações sobre o trânsito. Após, apresenta-se a proposta da aplicação do Smart Bus, o qual utiliza as tecnologias NFC e RFID para o monitoramento de dados sobre os transportes públicos, integrando um aplicativo para que possa ser efetuado o gerenciamento e a visualização das informações pelos usuários dos ônibus. Por fim, tem-se a discussão sobre as influências resultantes do compartilhamento de informação nos sistemas de transporte público, no que podem agregar em benefícios, nas implicações em relação à privacidade e à segurança dos dados de passageiros, além da infraestrutura tecnológica necessária à implantação de projetos envolvidos nos conceitos das Cidades Inteligentes e da Internet das Coisas. 


\section{As tecnologias de informação para a construção do transporte urbano inteligente}

Embora cada cidade possua sua própria cultura e particularidade, há desafios similares em grandes centros urbanos na área de transportes, como os congestionamentos no trânsito, os problemas com poluição, o transporte público ineficiente, a inadequação de vias, as dificuldades em obter informações de localização e outras situações correlatas, que trazem uma dinâmica de conflitos a serem explorados, a fim de que a sociedade possa refletir em busca de melhorias de funcionalidades (Braga, 2006; Toppeta, 2010; Batagan, 2011).

Nesse sentido, a gestão da infraestrutura urbana demanda competências e especializações que possam atender a um conjunto de fatores, expectativas e necessidades, oriundos de uma aceleração de crescimento que impacta a sociedade em proporções positivas e negativas. Com isso, o desenvolvimento depende de variados elementos, interligados e ao mesmo tempo independentes, mas cada vez mais imediatos. Planos e projetos são definidos em escalas de importância para funcionarem não apenas em longo prazo, mas também visando à solução de problemas pontuais e específicos de uma região. É o que aponta Ascher (2010), ao analisar o urbanismo como uma articulação de múltiplos projetos, com objetivos complementares em uma gestão estratégica.

Com a gama de processos que atua sobreposta em um sistema de transporte urbano, aumentam-se as dificuldades que podem ocorrer no funcionamento de sua logística por causa da baixa qualidade no compartilhamento de informações, como a falta de precisão das etapas de execução em que o sistema se encontra, os erros de relatórios, a demora no repasse de ocorrências, os dados não coincidentes com o panorama correto, entre outros. Esses distúrbios implicam em planejamentos que são executados sem a máxima eficiência que poderiam ter e que, por isso, comprometem o desempenho da gestão (Meyer et al., 2010).

É nessa complexidade que o desenvolvimento tecnológico pode atuar como um fator determinante para o crescimento do compartilhamento de informações, criando uma relação estreita entre as tecnologias digitais e os processos informacionais, proporcionando novas práticas comunicativas, redes de interações e diferentes produtos. Com isso, a tecnologia não apenas se insere na modificação do modo de vida das pessoas, em como consomem e produzem informações, mas também em como se relacionam entre si e com o meio (Atzori et al., 2010). Assim, constrói-se uma sociedade em rede, baseada na comunicação, na interação digital e no agrupamento de tecnologias, atuando na transformação estrutural e social (Castells, 2012). A partir dessa assimilação sobre as camadas de valor que a tecnologia pode oferecer à sociedade, surgem os conceitos das Cidades Inteligentes e da própria Internet das Coisas, atuantes na construção de sistemas de transporte urbano inteligentes.

\section{A relação entre as Cidades Inteligentes e a Internet das Coisas}

O conceito de Cidades Inteligentes encontra atuação na dinâmica entre as tecnologias de informação e comunicação (TICs) com os esforços organizacionais para implementar soluções inovadoras no gerenciamento das cidades (Toppeta, 2010). Para Harrison \& Donnelly (2011), as Cidades Inteligentes utilizam sistematicamente as TICs a fim de contribuir com a eficiência dos serviços urbanos. Com a mesma intenção, Dutta (2011) reconhece a importância das tecnologias para o suporte de questões determinantes nas cidades, como a competitividade da economia e a qualidade de vida geral da população. Portanto, entende-se que o papel das tecnologias é crucial para a viabilização das Cidades Inteligentes e para a consequente iniciativa de utilizar os recursos tecnológicos no gerenciamento dos sistemas urbanos (Toppeta, 2010; Batagan, 2011).

É nessa transformação que a chamada Internet das Coisas encontra crescimento, a partir de uma presença generalizada de uma variedade de coisas ou objetos capazes de interagir uns com os outros para alcançar objetivos comuns, oferecendo novas camadas de valor na sociedade (Sun, 2012; Guisto et al., 2010). Com a massiva utilização de objetos eletrônicos (como smartphones, tablets, televisores), integrados às redes de transmissão de dados (como GPS, Wi-Fi, Bluetooth, RFID e NFC), a tecnologia consolida-se como importante aliada do ser humano para a realização de tarefas diárias (Atzori et al., 2010). Conforme Sterling (2005), a implantação concreta da Internet das Coisas ocorre com o aumento de objetos que podem ser rastreados e que criam uma comunicação entre si. 
Dessa forma, pode-se entender o funcionamento da Internet das Coisas a partir de quaisquer itens que estejam ligados entre si, para a troca de informação e comunicação, a fim de identificar, localizar, rastrear, monitorar e gerenciar uma rede.

Nesse contexto, os Sistemas de Transporte Inteligente (Intelligent Transportation Systems) apoiam-se nos conceitos das Cidades Inteligentes e da Internet das Coisas, visando à aplicação de tecnologias de informação a veículos e infraestrutura, com o objetivo de ampliar a melhoria e a eficiência dos sistemas de transporte. As tecnologias envolvidas devem auxiliar, por exemplo, na redução de congestionamentos, mobilidade, segurança, poluição e produtividade nas vias (US DOT, 2009). Associado a isso, tecnologias de comunicação tornam-se cada vez mais como elementos integrantes no processo de soluções para o cenário do transporte urbano. Procuram-se alternativas visando à flexibilidade e à praticidade em cidades que enfrentam grandes problemas de trânsito.

Assim, tanto o NFC quanto o RFID apresentam-se como tecnologias capazes de agregar em conexão, acesso e transações financeiras nos estágios antes, durante e após as jornadas dos trajetos. Os benefícios com as tecnologias passam por informações sobre o transporte da cidade, tais como: mapas e pontos de localização, horários de trens, metrôs e ônibus, tráfego em tempo real, telefones úteis, serviços de táxis. Além disso, há as funcionalidades dos acessos às catracas e da compra de bilhetes (NFC Fórum, 2011).

Apresenta-se a seguir as definições e o funcionamento das tecnologias RFID e NFC, a fim de melhor compreendê-las e, posteriormente, contextualizá-las como integrantes das Cidades Inteligentes e que podem auxiliar o transporte urbano.

\section{O funcionamento do RFID}

A tecnologia RFID é uma rede de comunicação a distância sem fio, que funciona pela identificação de frequências de rádio. 0 alcance pode chegar a metros de distância, dependendo do tipo de chip e antena utilizados. A comunicação ocorre por meio de uma etiqueta com chip RFID, a chamada Tag RFID, que envia sinais a um leitor específico. A partir disso, um software é responsável pela conversão dos dados em informações significativas (Duroc \& Kaddour, 2012).
Assim, a tecnologia RFID pode ser utilizada com o objetivo de identificação ou rastreamento de objetos, aplicações do setor logístico, de supermercados, de transporte ou de cargas. Apenas é necessário que o produto possua a etiqueta com RFID para que os dados possam ser capturados pelo leitor, mesmo que os produtos estejam em movimento (Sun, 2012).

Há dois modos de funcionamento de uma Tag RFID:

1) Ativo: a Tag possui uma fonte de alimentação externa, o alcance de comunicação é variável em metros e é capaz de enviar dados a um leitor por conta própria.

2) Passivo: neste modo, não há bateria e a corrente é fornecida pelo leitor. Uma Tag RFID passiva possui um alcance de leitura com uma distância menor e normalmente também possui um tamanho menor, se comparado a uma Tag ativa.

Nesse sentido, a rastreabilidade de elementos realizada pela tecnologia RFID é um dos elementos aplicados em processos a fim de fornecer dados para melhor análise e gestão da informação. Entre os benefícios adquiridos com a utilização do RFID, destacam-se: ganho de visibilidade na logística (Angeles, 2006), durabilidade das etiquetas (Freiberger \& Bezerra, 2010; Soares et al., 2008), identificação sem a necessidade de contato ou visão do produto (Freiberger \& Bezerra, 2010; Navarro et al., 2008), confiança na transmissão dos dados e na leitura de múltiplas etiquetas simultaneamente (Navarro et al., 2008; Rosa, 2006), redução de tempo de leitura (Costa et al., 2010; Soares et al., 2008), captação de ondas a distância, rastreabilidade de produtos e de informações (Navarro et al., 2008), captura de dados de produtos que estão em movimento e em ambientes agressivos (Rosa, 2006).

\section{O funcionamento do NFC}

O NFC é uma tecnologia que permite a transferência de dados em uma comunicação sem fio de curta distância, por aproximação. O NFC surgiu a partir do RFID, foi desenvolvido pela Sony e Philips em 2002, e impulsionado a partir de 2004 pelo NFC Forum, que é conduzido por empresas como Samsung, Microsoft, Nokia, Google, Intel e Visa (NFC Fórum, 2011). Com o suporte de empresas de grande porte no mercado tecnológico, cada vez mais aparelhos eletrônicos 
possuem NFC, como smartphones de diferentes categorias da Motorola, LG e Samsung, consideradas como as marcas com maior participação no mercado brasileiro de telefonia móvel.

Assim como o RFID, há transmissão de dados por radiofrequência, mas a comunicação ocorre apenas pela aproximação dos dispositivos com NFC em uma curta distância. Dessa forma, os usuários podem realizar transações financeiras, acessar catracas e conectar diferentes aparelhos para compartilhamento de informações (Ozdenizci et al., 2010). Os chips também podem vir tanto em smartphones e eletrônicos quanto em etiquetas (Tags NFC/RFID). Com isso, é possível compartilhar informações entre diferentes objetos, como crachás, chaveiros e cartões.

Há dois modos de funcionamento do NFC:

1) Ativo: neste modo, os dois dispositivos envolvidos na comunicação podem realizar transferências de dados, enviando e recebendo. 0 modo ativo ocorre, por exemplo, entre smartphones para troca de arquivos de mídias, como fotos e vídeos.

2) Passivo: neste comportamento, um dos dispositivos emite um sinal, enquanto o outro apenas recebe a informação, estabelecendo uma comunicação de uma via. É o caso de um smartphone que recebe os dados de uma Tag NFC ou na transmissão de um cartão para acesso a uma catraca (NFC Fórum, 2011).

\section{Aplicação de tecnologias de informação para auxílio a sistemas de transporte urbano inteligente}

Nesta etapa, objetivou-se apresentar como diferentes cidades brasileiras e no mundo encontram soluções em tecnologias para auxiliar seus sistemas de transporte urbano e aplicar os conceitos de Cidades Inteligentes e Internet das Coisas. Assim, pode-se ter uma visão mais clara sobre as soluções apresentadas para diferentes problemas da cidade no que se refere ao trânsito e ao transporte público.

Em um primeiro momento, destacam-se as aplicações que utilizam as tecnologias NFC e RFID. Em seguida, há o objetivo de mostrar como variados aplicativos móveis funcionam para gerenciar as informações de trânsito.

\section{Aplicação de RFID e NFC no transporte urbano}

Já há alguns anos, o conceito das Cidades Inteligentes é materializado em soluções que aplicam as tecnologias NFC e RFID para auxiliar sistemas de transporte urbano. São listadas as aplicações a seguir.

NFC em programa de ônibus Roda SP

O projeto Roda SP é realizado durante o verão em São Paulo e oferece a funcionalidade de transitar 24 horas pelos municípios da Baixada Santista com uma única passagem de ônibus. Assim, o passageiro pode embarcar e desembarcar entre os 32 pontos participantes quantas vezes desejar. 0 controle era realizado até 2012 com uma pulseira simples no braço, o que dificultava o controle da Secretaria de Turismo.

Em 2013, o programa testou o Cartão de Acesso NFC, desenvolvido pela instituição CPqD (Centro de Pesquisa e Desenvolvimento em Telecomunicações). Ao comprar a passagem, o usuário recebia um cartão NFC, que era utilizado em cada embarque e desembarque dos ônibus. Ao utilizar o cartão no primeiro embarque, as 24 horas de uso começavam a contar. A aplicação foi feita associada a smartphones presentes nos veículos, e não em catracas. Dessa forma, o sistema com NFC contribuiu com a logística do Roda SP e com os relatórios da Secretaria de Turismo, que teve o controle exato do número de passageiros que estiveram nos ônibus, os pontos com maior fluxo e os períodos mais intensos de utilização (NFC Brasil, 2013).

RFID em acesso a pedágios no Brasil

Desde 2000, algumas cidades no Brasil possuem o sistema de pedágio Sem Parar/Via Fácil que possibilita o acesso inteligente de veículos por catracas nas estradas e também em estacionamentos. O projeto funciona com o usuário fixando a Tag RFID no automóvel. Ao se aproximar do local do pedágio, uma antena RFID próxima à cancela identifica a Tag e libera a passagem, sem a necessidade de parar o carro para realizar o pagamento. Ao final do mês, o usuário recebe o boleto com o valor dos pedágios. Em 2013, o sistema sofreu algumas modificações com o intuito de tornar o valor do pedágio segmentado de 
acordo com a quantidade de quilômetros trafegados (RFID Journal, 2013a).

0 sistema Ponto a Ponto funciona de maneira similar ao Sem Parar, mas com o modelo de pré-pagamento. Assim, o usuário compra créditos que são descontados conforme a utilização dos pedágios. 0 programa também instalou pontos com leitores RFID pelas estradas, os quais funcionam para identificar as etiquetas dos carros, para que a cobrança seja feita de acordo com a distância percorrida e sem a necessidade de catracas (RFID Journal, 2013b).

Pagamentos com NFC na Alemanha, Estados Unidos e Inglaterra

Desde 2007, são realizados testes para a realização de pagamentos com NFC substituindo os tradicionais bilhetes de passagem utilizados em transporte público. Em cidades da Alemanha, o projeto Touch\&Travel testou com aproximadamente 3 mil participantes a aceitação do NFC em smartphones como meio de acesso para o embarque e desembarque em trens. Os benefícios informados foram a flexibilidade para a emissão de bilhetes, com baixa infraestrutura e custo.

Nos Estados Unidos, uma parceria entre o sistema de trânsito de São Francisco e empresas de fast-food resultou em um projeto que possibilitava o pagamento móvel com NFC no transporte público e ainda a compra de refeições nos estabelecimentos parceiros por meio de smart posters presentes nas estações de embarque.

Em Londres, também foi testado um projeto que utilizava smartphones com NFC para compra de bilhetes e acesso, em substituição aos cartões de transporte tradicionais. Como resultado, os participantes demonstraram alto nível de interesse e satisfação no projeto pela conveniência, facilidade de uso e status que sentiram com a aplicação (NFC Fórum, 2011).

\section{Aplicativos de auxílio ao transporte urbano}

Consultas de ônibus

Em São Paulo, há o projeto Olho Vivo, desenvolvido pela SPTrans e disponível ao público desde 2008. O sistema funciona por meio de aparelhos GPS instalados nos ônibus da cidade e com os dados integrados ao Google Maps (Figura 1). Acessando a versão desktop ou o aplicativo "Onde Está Meu Ônibus?" para smartphones, os usuários podem consultar o trajeto percorrido pelos veículos, com a localização no mapa, a velocidade média e os horários previstos para estarem nos pontos (SPTrans, 2016). Contudo, a informação em tempo real não cruza os dados com os pontos de forma precisa, uma vez que as localizações e os horários fornecidos ocorrem por aproximação das coordenadas virtuais de ônibus e de pontos no mapa, e não pela marcação física nas vias de circulação. 0 usuário também necessita de acesso à internet para poder obter a atualização dos dados de localização dos veículos.

Outros aplicativos também buscam auxiliar os usuários de transporte público nas cidades, oferecendo informações gerais para consulta das linhas, horários previstos, trajetos e pontos em que passam os ônibus. É o caso dos aplicativos WBus (Figura 2), Próximo Ônibus e Buzzão, que operam com funções similares. Embora forneçam dados do transporte coletivo, não há uma atualização em tempo real de forma automática sobre horários e localizações, mas uma estimativa de acordo com as informações fornecidas pelas empresas prestadoras do serviço e pelos órgãos responsáveis pelo gerenciamento do transporte nas cidades (WBus, 2016).

\section{Mapa colaborativo}

Com o objetivo de agregar o poder da mobilização popular a um sistema de monitoramento, o aplicativo para smartphones Waze trabalha com dados fornecidos pelos próprios usuários. Funcionando em diversas cidades do Brasil e do mundo, as atualizações podem ser cadastradas por qualquer indivíduo. Assim, cria-se um mapa colaborativo com relatos sobre acidentes, manifestações de ruas, horários e localizações de transportes públicos, vias que estão congestionadas ou com problemas de acessos, entre outras informações (Figura 3). Com isso, o banco de dados é atualizado conforme a atuação dos usuários, com as dicas e as opiniões (Waze, 2016). Para que tenha acesso aos dados atualizados em tempo real no aplicativo, o usuário deve possuir conexão com internet no smartphone.

De modo similar, o aplicativo Moovit adota o compartilhamento das informações fornecidas pela comunidade de usuários com o objetivo de abastecer um mapa colaborativo com dados sobre atrasos dos 


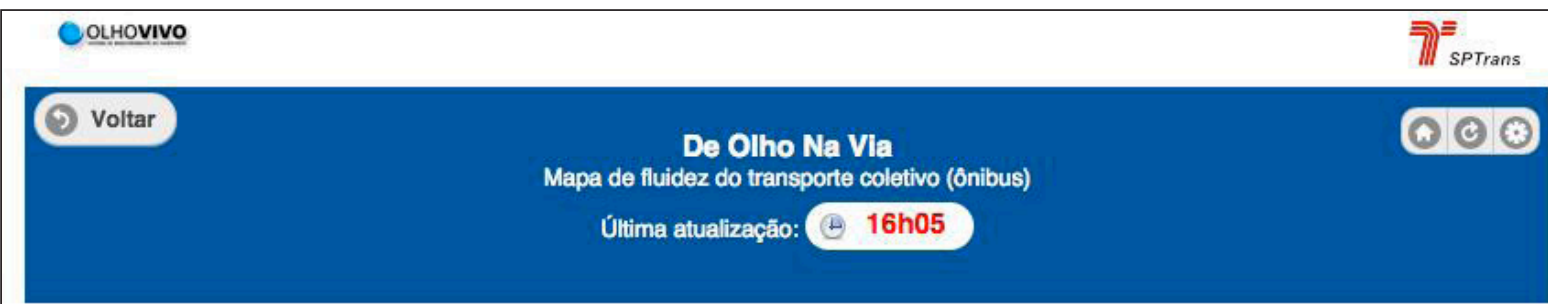

Digite seu Endereço

OK

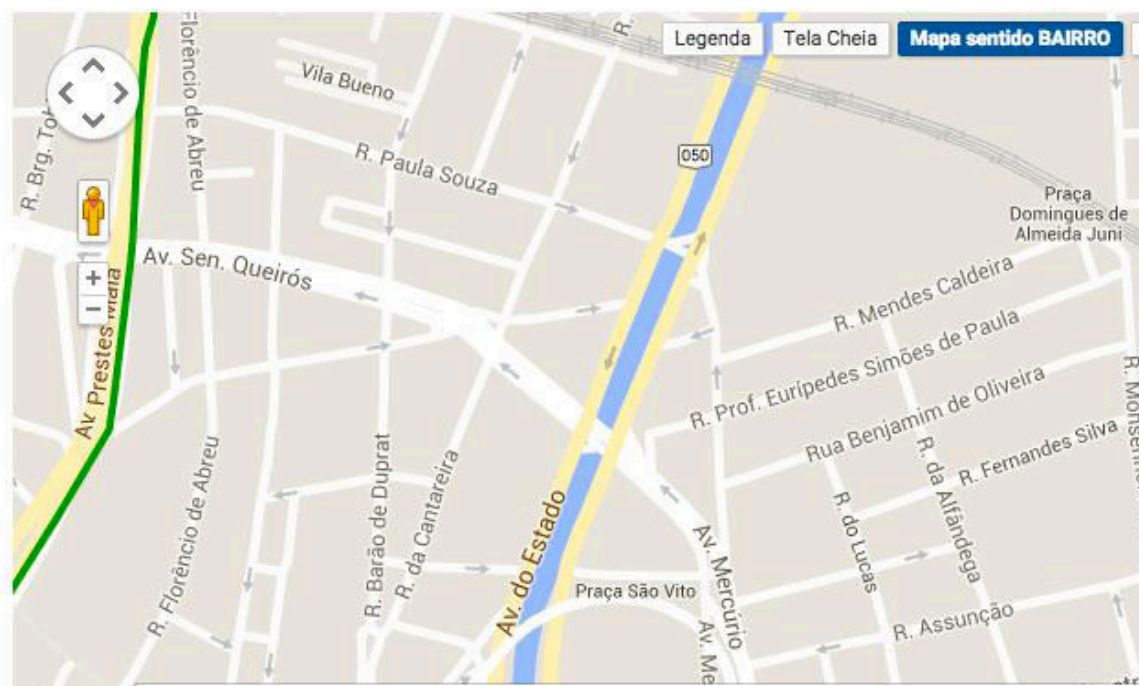

Mapa sentido CENTRO

Mapa $\quad-$

CELSO GARCIA (CENTRO - BAIRRO)

Trecho: de R.FIGUEIRA(BC) / VD.25 DE MARÇO (CB) até R.MONS. ANACLETO (BC)/R. CARNEIRO LEÃO (CB) Referência: R. GASOMETRO(BC)/AV. RANGEL PESTANA(CB) Extensão (metros): 550

Velocidade média do trecho

Tempo médio do percurso

$13 \mathrm{~km} / \mathrm{h}$

OOh02

Velocidade média da via: $18 \mathrm{~km} / \mathrm{h}$

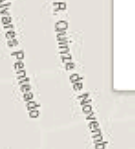

unifacio

$\rightarrow \quad$

ijo $e^{5^{\circ}}$

10

'raça Dr.
io Mendes

Mendes Largo Sete
de Setembro

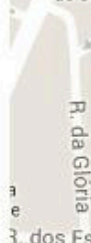

7. dos Estudantes
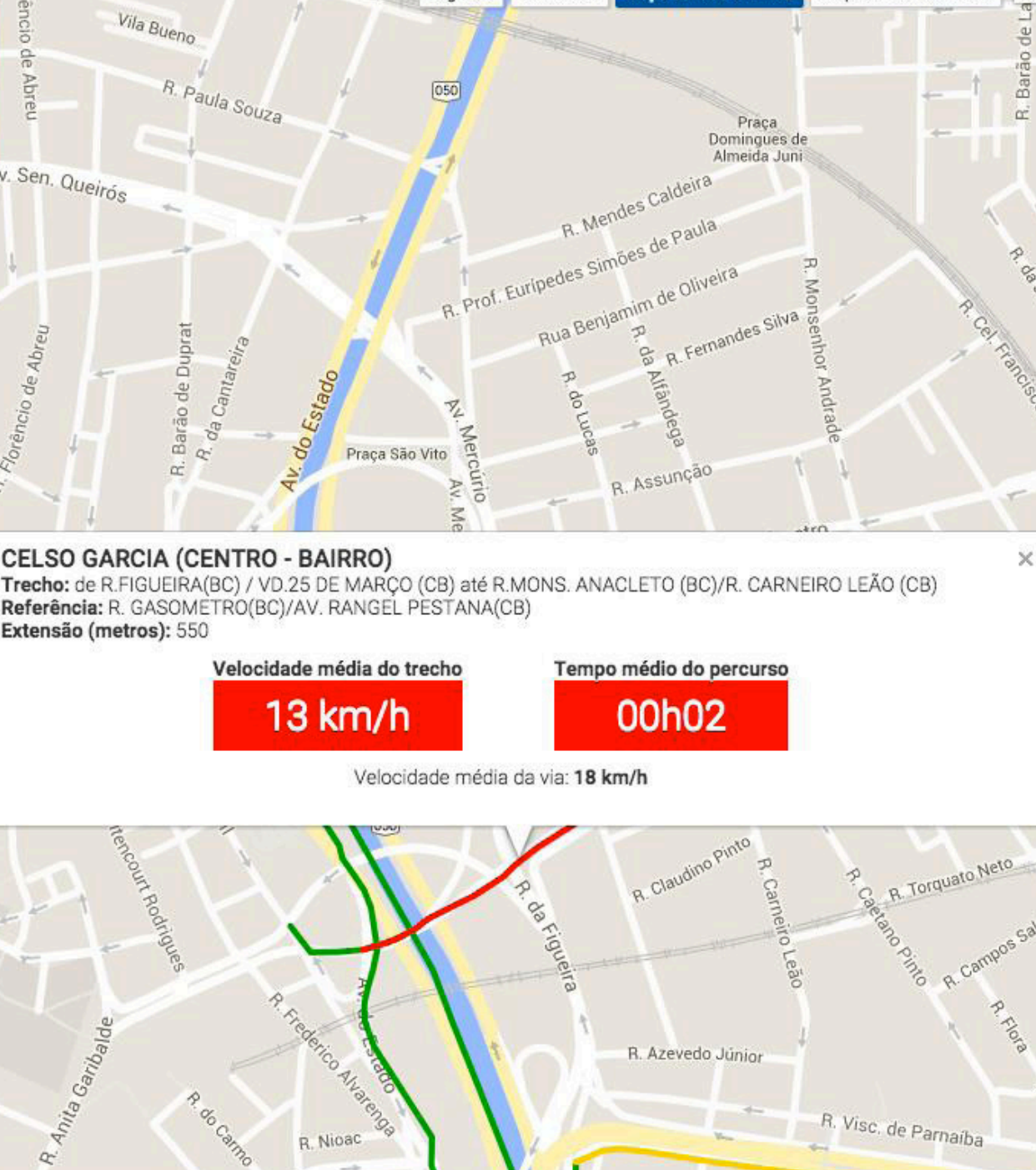

일

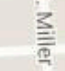




\begin{tabular}{|c|c|c|}
\hline †े (2) 새 & D $3 \mathbb{N}\}$ & 展 4 (1) 21:2 \\
\hline WBus Lite & & \\
\hline$\sum_{0.00}^{\text {WBus }} \mathrm{Hc}$ & ários & \\
\hline Linha: 0130 & orte//ririú/Tu & \\
\hline Fonte: Tran & & \\
\hline Saida: Term & I Norte $\mathrm{p} / \mathrm{T \epsilon}$ & nal Iririú \\
\hline Dias Úteis & Sábados & Domingos \\
\hline 20:00 & $04: 15$ & e feriados \\
\hline $20: 25$ & 04:38 & $05: 18$ \\
\hline $20: 50$ & 05:05 & $06: 25$ \\
\hline $21: 15$ & $05: 30$ & 07:10 \\
\hline $21: 40$ & $05: 55$ & $07: 55$ \\
\hline $22: 05$ & $06: 20$ & 08:40 \\
\hline $22: 22$ & $06: 45$ & $09: 25$ \\
\hline $22: 30$ & 07:10 & $10: 10$ \\
\hline $22: 55$ & $07: 35$ & $10: 55$ \\
\hline 23:20 & ח ח & $11: 40$ \\
\hline
\end{tabular}

Figura 2 - Aplicativo WBus Fonte: WBus (2016).

ônibus, qualidade e defeitos dos serviços de transporte, e possibilidade de qualificar o desempenho dos motoristas de ônibus e táxis (Figura 4). Além disso, o Moovit possui as rotas e a duração dos trajetos de trens, metrôs e ônibus de variadas cidades. 0 aplicativo funciona por meio de rede de conexão com a internet e ativação do GPS no smartphone a fim de poder atualizar os dados em tempo real e gerar alternativas de caminhos para o usuário, depois de uma seleção de origem e destino pretendidos (Moovit, 2016).

\section{Proposta de aplicação: Smart Bus}

Após análise de diferentes aplicações de tecnologias de informação e de conectividade ao transporte urbano, propõe-se uma integração de funcionalidades em um único projeto. Trata-se da aplicação do Smart Bus, que visa ao gerenciamento de dados sobre o transporte público nas cidades e à visualização das informações

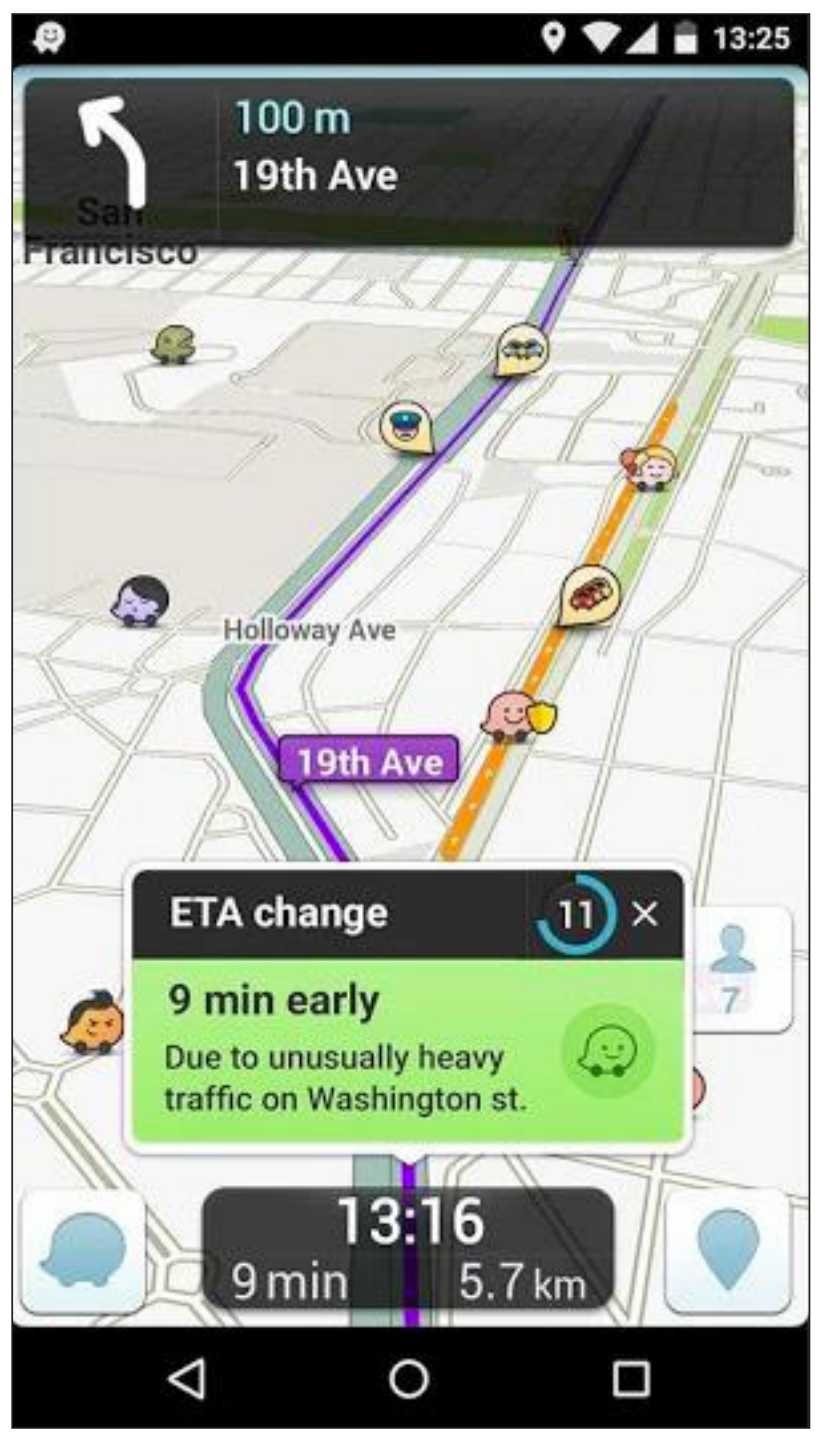

Figura 3 - Aplicativo Waze

Fonte: Waze (2016).

pelos usuários dos ônibus. A proposta do Smart Bus consiste na instalação de aparelhos com NFC/RFID (sistema de antena/leitor para transmissão dos dados) nos terminais de ônibus e dentro dos próprios veículos, além de um aplicativo para que se possa estabelecer a gestão do sistema e a consulta aos dados gerados.

Inicialmente, o usuário deve possuir o aplicativo Smart Bus em seu smartphone. Com o download, a base de dados é obtida, possibilitando a consulta e posterior utilização de modo off-line. Assim, não há a necessidade de acesso à internet para o funcionamento, uma vez que a troca de informações é efetuada pelas tecnologias de radiofrequência NFC/RFID presentes nos smartphones e nos aparelhos instalados nos ônibus e pontos. 0 acesso à internet é necessário apenas para 


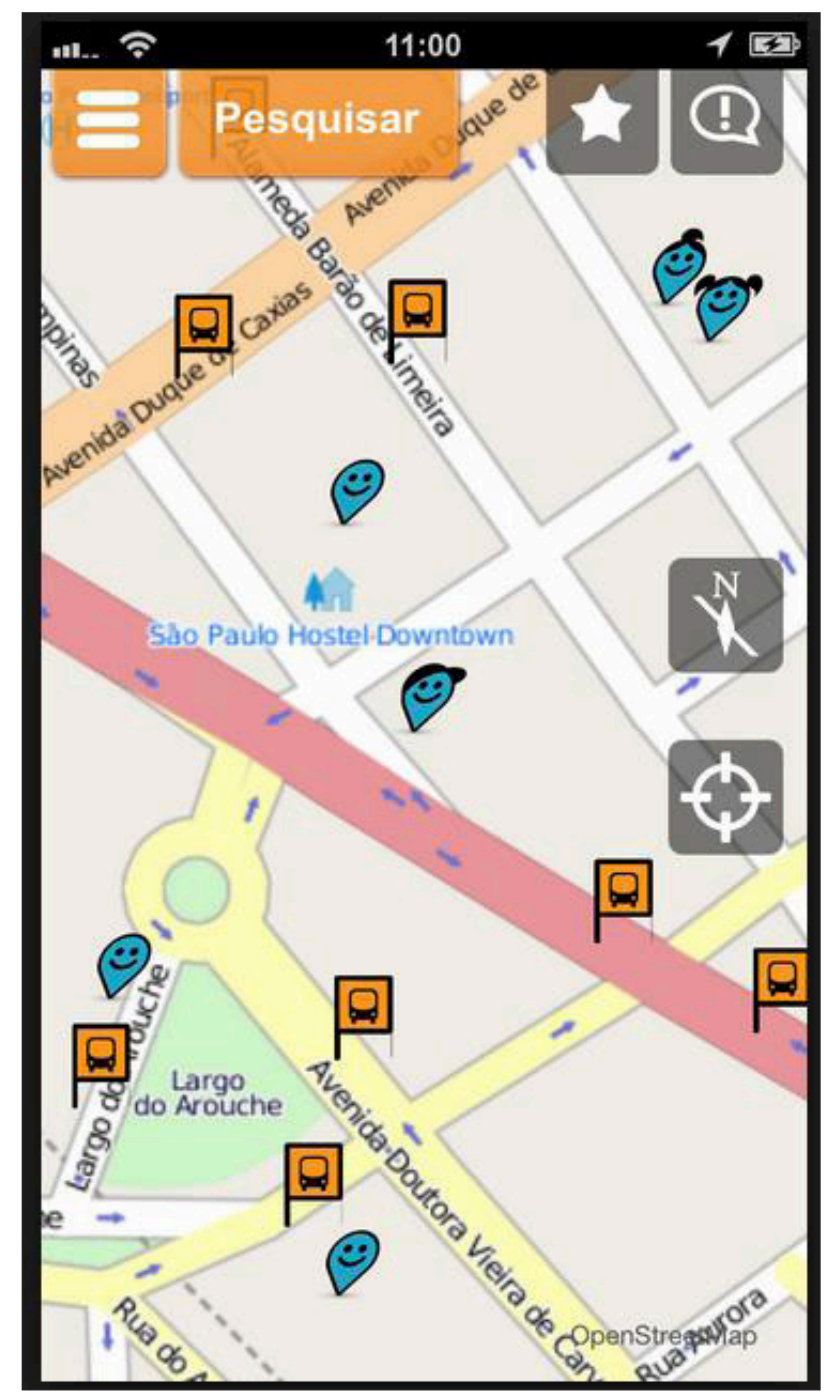

Figura 4 - Aplicativo Moovit

Fonte: Moovit (2016).

a atualização do aplicativo, conforme novas rotas, linhas de ônibus e demais informações quando forem cadastradas ou alteradas, além de possibilidade de atualização de versão do próprio aplicativo.

A partir disso, o funcionamento do sistema ocorre pela transmissão de dados efetuada todas as vezes em que o ônibus transitar pelo ponto ou terminal e pela aproximação do celular com NFC dos aparelhos instalados nos ônibus ou pontos. Com isso, o usuário poderá realizar uma série de consultas atualizadas sobre as linhas e os pontos de ônibus da cidade, como horários em que passaram nos pontos, além dos endereços por onde os ônibus trafegam.

Seguem as funcionalidades da aplicação:

- Consulta das linhas de ônibus que passam nos pontos da cidade;
- Consulta dos horários em que as linhas de ônibus passaram nos pontos;

- Consulta dos endereços dos pontos e terminais em que a linha passa;

- Gerenciamento de dados para as empresas de ônibus, em virtude da marcação automática e precisa do horário em que a linha de ônibus passou nos pontos;

- Gerenciamento de dados para o setor público, com informações precisas sobre o desempenho do transporte coletivo.

\section{Funcionamento do Smart Bus}

O processo de execução do Smart Bus é estabelecido em três momentos:

1) Passagem do ônibus pelo ponto ou terminal;

2) Acesso aos dados no ponto;

3) Acesso aos dados no ônibus.

As etapas são descritas a seguir, com as respectivas ilustrações.

Comunicação entre ônibus e ponto/terminal

Tanto os ônibus quanto os pontos possuem aparelhos com a tecnologia RFID/NFC (Figura 5). Quando o ônibus passa pelo ponto, ocorre a troca de informações entre os aparelhos. Ficam registradas no aparelho do ponto a hora e a respectiva linha de ônibus. Já no aparelho do ônibus, ficam registrados os horários com os respectivos pontos pelos quais passou.

\section{Comunicação entre usuário e ponto}

Quando o usuário aproxima seu celular com NFC ao aparelho do ponto de ônibus (Figura 6), recebe as informações com todas as linhas de ônibus e suas respectivas horas previstas para passarem naquele ponto. Da mesma forma, é informado também o histórico de linhas e de horários em que já passaram (Figura 7). Com isso, o passageiro pode saber se seu 


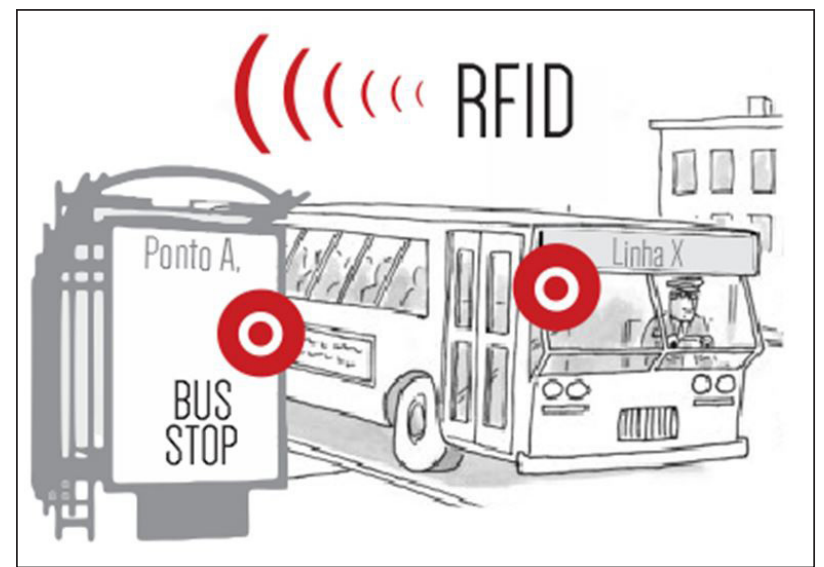

Figura 5 - Ônibus envia e recebe dados com o ponto Fonte: Os autores.

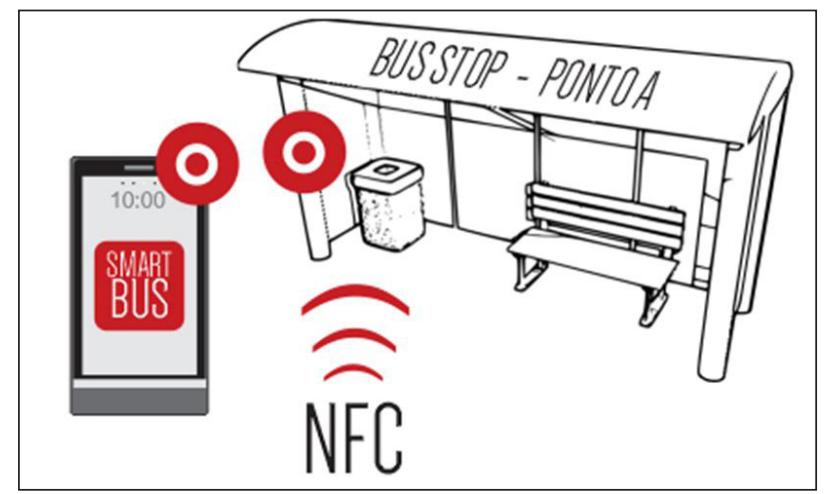

Figura 6 - Usuário recebe dados do ponto no smartphone Fonte: Os autores.

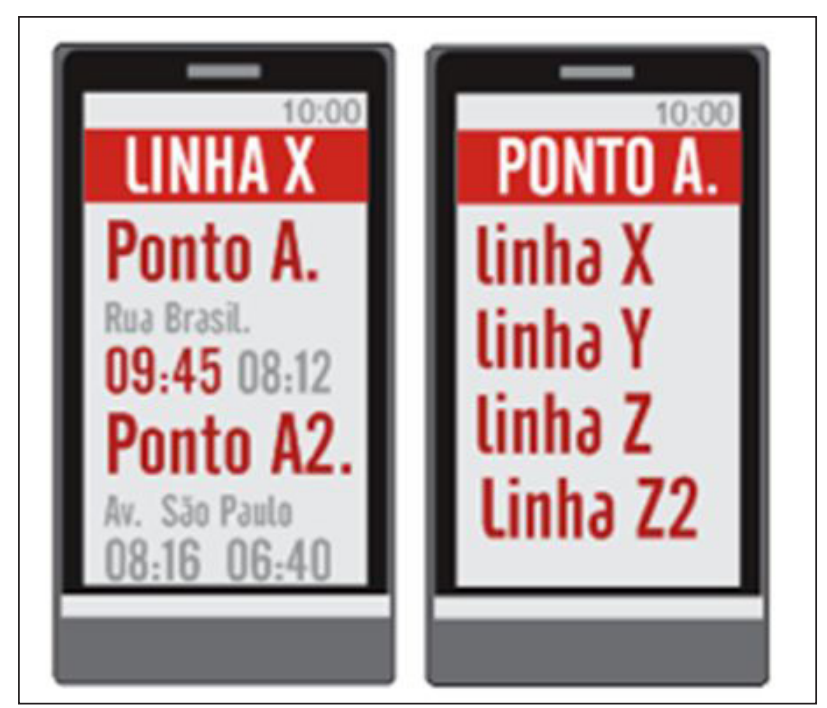

Figura 7 - Consulta no ponto de ônibus Fonte: Os autores. ônibus já passou ou em quais outros ônibus poderia embarcar de acordo com a hora.

Outra forma de consulta ocorre pelo acesso detalhado sobre cada uma das linhas de ônibus que passam pelo ponto (Figura 7). Assim, o usuário também pode se informar sobre os pontos e os endereços em que a determinada linha de ônibus trafega. Desse modo, o passageiro sabe se o ônibus que pretende utilizar irá passar no destino pretendido ou então saber qual ônibus correto deve utilizar, de acordo com o endereço em que deve chegar.

Comunicação entre usuário e ônibus

Uma vez embarcado em uma linha de ônibus, também há a possibilidade de compartilhamento de informação entre o smartphone do usuário e o aparelho do ônibus (Figura 8). Ao aproximar o celular com NFC ao aparelho presente internamente no veículo, o usuário recebe a informação com todos os horários e os pontos em que aquela linha passou, bem como as próximas paradas nos pontos ou terminais. Além disso, o passageiro tem o endereço correto de cada ponto em que o ônibus passou. Dessa forma, se tem um histórico preciso para a consulta.

Também é possível realizar uma consulta detalhada sobre quais os pontos e os terminais aquele ônibus trafega, conhecendo as outras linhas que param naqueles locais (Figura 9). Assim, o passageiro pode obter a informação precisa sobre quais são os outros ônibus que passam nos pontos em que o seu ônibus para (Figura 9). Dessa forma, auxilia os passageiros que precisam trocar de ônibus para chegarem até o seu destino.

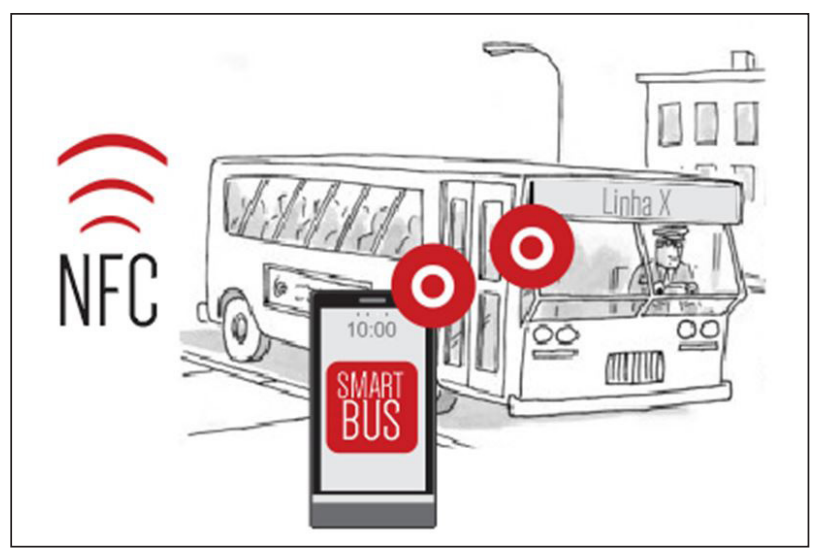

Figura 8 - Usuário recebe dados da linha de ônibus no seu smartphone Fonte: Os autores. 


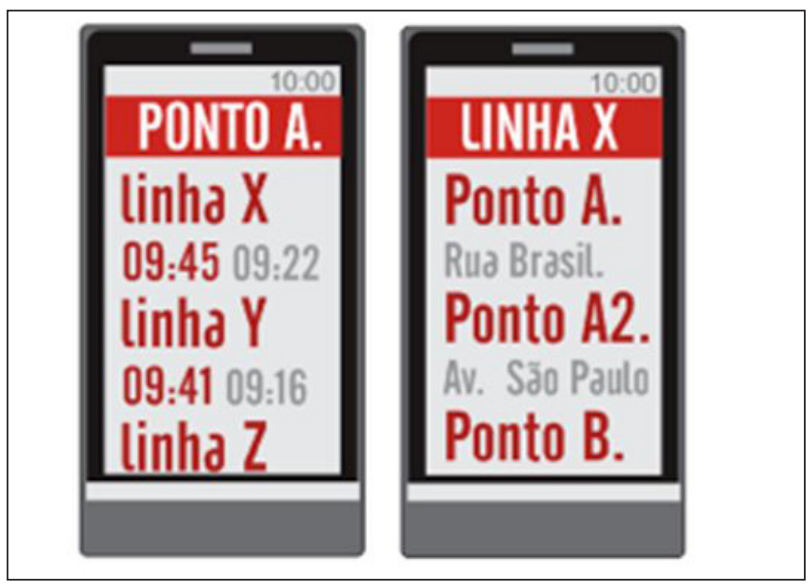

Figura 9 - Consulta na linha de ônibus

Fonte: Os autores.

\section{Discussão sobre o compartilhamento de informações no transporte público}

A utilização de tecnologias que objetivam auxiliar um problema específico da sociedade reflete o caráter do desenvolvimento alcançado nos últimos anos. Aos poucos, forma-se uma base de infraestrutura, conhecimento e aplicações capaz de servir às necessidades das pessoas nas grandes cidades. Nesse contexto, a aplicação do Smart Bus trata não apenas de incluir o RFID e o NFC como diferenciais de interação, mas com o objetivo de visualizar em como podem agregar em termos de facilidade para quem utiliza os serviços de ônibus e para as organizações atuantes no transporte público.

De acordo com o US DOT (2009), pode-se inferir que uma proposta como o Smart Bus tem potencial para configurar o chamado Sistema de Transporte Inteligente (Intelligent Transportation System), pois visa à melhoria e à eficiência dos sistemas de transporte, contribuindo com dados e informações à população, além da precisão e maior controle para as organizações atuantes no setor de transporte urbano, como a secretaria de transportes e as empresas privadas de transporte coletivo, que passam a obter mais fontes capazes de auxiliar no próprio gerenciamento do setor.

A partir de um sistema que conecta ônibus públicos a pontos e terminais, há a possibilidade de se realizar um mapeamento do perfil de vias com os dados de horários em que os ônibus passam nos pontos, o tempo que os ônibus levam para trafegar de um ponto ao outro, os períodos e os locais em que há maior lotação nos pontos e veículos, localizando áreas em que ocorrem os maiores atrasos em virtude de congestionamento e dificuldades de acesso. Assim, é possível detectar com precisão essas problemáticas e promover modificações que atentem à produtividade nas vias.

Outro aspecto a ser mencionado é do incentivo à utilização do transporte público pela geração de conhecimento. Como muitas pessoas deixam de utilizar ônibus por não terem a informação sobre qual linha passaria em um destino pretendido, o Smart Bus poderia promover maior confiança a esses usuários. Com a mensuração dos dados proporcionados pela tecnologia, pode-se ter noção das áreas em que houve aumento da utilização do transporte público, cruzando dados com o acesso dos passageiros ao aplicativo, resultado do maior conhecimento sobre as vias.

Portanto, visualiza-se o Smart Bus como uma ferramenta consistente de aplicação das tecnologias de informação e da Internet das Coisas, tornando-se um elemento integrante no processo de soluções para o cenário do transporte urbano. Observa-se a utilização de RFID e NFC como tecnologias capazes de sustentar a flexibilidade e a praticidade em cidades que enfrentam grandes problemas de trânsito, agregando em conexão e acesso a informações nos estágios antes, durante e após as jornadas dos passageiros. Como não requer acesso à internet para a atualização dos dados sobre horários em que os ônibus passaram, e a base de consulta pode ser utilizada de modo off-line, pode-se ampliar a faixa de público a ser atingida, com maior possibilidade de utilização para os usuários do transporte público que não possuem acesso à internet ou que possuem acesso limitado de dados.

Há de se ressaltar também os aspectos relacionados à privacidade e à segurança dos dados de passageiros. Embora o compartilhamento de informações sobre o transporte público possa agregar benefícios aos usuários, também há a preocupação em torno da proteção dos dados dos indivíduos, uma vez que há uma conectividade estabelecida entre o smartphone e os aparelhos NFC/RFID presentes nos pontos/terminais e nos ônibus.

O sistema NFC/RFID em si pode possuir criptografia para assegurar a proteção dos dados. Mas, como um fator envolvido no próprio contexto da Internet das Coisas, é possível questionar o monitoramento exercido sobre os usuários pelas organizações que efetuam a gestão do sistema, a partir da criação de um histórico de trajetos, linhas e locais frequentados, com respectivo 
registro de dias e horários em que as atividades são realizadas. Conforme destacam Coskun et al. (2013), são situações pertinentes à própria existência dos aparatos tecnológicos, sujeitos a utilizações maliciosas e com fins prejudiciais, assim como a vulnerabilidade de aplicações na internet e em dispositivos móveis. É uma discussão que não se restringe a determinado projeto ou tecnologia aplicada, mas que permeia o conceito de Cidades Inteligentes, uma vez que toda manifestação em torno do meio tecnológico depende da intenção de utilização dos usuários. Cabe não apenas à ética das organizações envolvidas em serviços urbanos, mas também à fiscalização das agências reguladores e à implementação de leis que ofereçam o suporte necessário para garantir a segurança dos dados dos usuários.

A disseminação de projetos que contemplem o compartilhamento de informações entre pessoas e as tecnologias implica em novos desafios e discussões na sociedade sobre os aspectos legais e filosóficos dessas transformações. Qualquer produto disponibilizado ao cidadão está sob regime das leis de um país. No caso da aplicação do Smart Bus, os dados obtidos com a interação nos pontos de ônibus e internamente nos veículos podem ser autorizados pelos próprios usuários no momento em que se efetua o download do aplicativo. Mas, no meio digital em geral, não há clareza sobre a possibilidade de utilização dos dados fornecidos, sobre o que pode ser compartilhado por aplicativos, o tipo de monitoramento efetuado com o usuário de determinado serviço ou por quanto tempo pode-se e/ou deve-se armazenar as informações. Apesar de oferecer benefícios por meio da utilização dos dados, como o gerenciamento das linhas de transporte público, muito há a se discutir a respeito da moralidade existente no compartilhamento de informações na sociedade como um todo, o que merece um aprofundamento em um trabalho específico para tanto.

Por fim, para efetivar uma aplicação como o Smart Bus, ressalta-se a necessidade de investimento em infraestrutura de ônibus e terminais a fim de instalar os aparelhos NFC/RFID e possibilitar a rede de comunicação e o compartilhamento de informações atualizadas sobre horários e linhas. Essa ampliação da capacidade tecnológica disponível nos centros urbanos é um dos fatores decisivos para tornar possível o desenvolvimento das Cidades Inteligentes, já reforçada por Toppeta (2010) e Batagan (2011), como aumento da disponibilização de pontos de acesso gratuito à internet, fontes de alimentação de energia e disseminação de redes de conectividade. A própria gestão sobre o funcionamento da aplicação requer esforço para a divulgação sobre as tecnologias NFC/RFID, que não contam com familiarização da sociedade, ainda que estejam presentes em diversos aparelhos eletrônicos domésticos e inclusive em projetos relacionados ao transporte urbano.

\section{Considerações finais}

O objetivo deste artigo foi o de analisar como as tecnologias de informação podem auxiliar sistemas de transporte urbano apoiando-se no contexto das Cidades Inteligentes e da Internet das Coisas. De modo específico, delimitou-se o tipo das tecnologias em RFID e NFC, e em como podem atuar no compartilhamento de informações do transporte público. Após o levantamento bibliográfico sobre os conceitos envolvidos e diferentes projetos aplicados aos sistemas de transporte, propôs-se a aplicação do Smart Bus com o intuito de integrar em um único projeto as tecnologias RFID e NFC, associadas a um aplicativo, para atuar no compartilhamento de informações sobre o transporte público.

A aplicação do Smart Bus visa ao gerenciamento de dados sobre o transporte público nas cidades e à visualização das informações pelos usuários dos ônibus. Com as três etapas de funcionamento do Smart Bus (1-troca de dados entre ônibus e ponto, 2-consulta do usuário no ponto e 3-consulta do usuário no ônibus), verificaram-se as contribuições no que diz respeito ao acesso a informações, monitoramento e controle de dados. Observa-se que, a partir da aplicação, pode-se mapear um perfil das vias, ônibus e passageiros, localizando atrasos e maior volume do tráfego de linhas. Com isso, geram-se fontes de dados para detectar problemas e promover melhorias ao transporte público. A partir da mensuração da utilização do Smart Bus pelos passageiros, obtêm-se informações relevantes sobre horários e locais de maior utilização, além do incentivo à utilização do transporte público, em virtude do maior conhecimento sobre as linhas e os endereços.

A partir da aplicação proposta do Smart Bus, discutiu-se o papel do compartilhamento de informações no transporte público, em como pode agregar em funcionalidades para quem utiliza os 
serviços de ônibus e para as organizações atuantes no setor. Discutiu-se ainda sobre os aspectos que permeiam os conceitos das aplicações atuantes no meio da Internet das Coisas, no que diz respeito à privacidade e à segurança dos dados de passageiros, bem como às implicações para a formulação de leis de proteção aos usuários. Além disso, pôde-se ressaltar a necessidade da ampliação de uma infraestrutura tecnológica nos centros urbanos a fim de tornar viável não apenas o compartilhamento de informações, mas o desenvolvimento das Cidades Inteligentes.

Por fim, espera-se que o artigo tenha oferecido contribuição para a reflexão de como tecnologias de transmissão de dados a distância, como NFC e RFID, podem auxiliar o gerenciamento dos sistemas de transporte urbano como um todo, proporcionando soluções em diferentes escalas e necessidades por meio do compartilhamento das informações pelos usuários e organizações atuantes no setor.

\section{Referências}

Angeles, R. (2006). RFID technologies: supply-chain applications and implementation issues. Information Systems Management, 22(1), 51-65. http://dx.doi.org/10 .1201/1078/44912.22.1.20051201/85739.7.

Araújo, M. R. M., Oliveira, J. M., Jesus, M. S., Sá, N. R., Santos, P. A. C., \& Lima, T. C. (2011). Transporte público coletivo: discutindo acessibilidade, mobilidade e qualidade de vida. Psicologia e Sociedade, 23(2), 574-582. http://dx.doi. org/10.1590/S0102-71822011000300015.

Ascher, F. (2010). Os novos princípios do urbanismo. São Paulo: Romano Guerra.

Atzori, L., Iera, A., \& Morabito, G. (2010). The internet of things: a survey. Computer Networks, 54(15), 2787-2805. http://dx.doi.org/10.1016/j.comnet.2010.05.010.

Batagan, L. (2011). Smart cities and sustainability models. Informatica Economica, 15(3), 80-87.

Braga, T. M. (2006). Sustentabilidade e condições de vida em áreas urbanas: medidas e determinantes em duas regiões metropolitanas brasileiras. Revista Eure, 32(96), 47-71.

Castells, M. (2012). A sociedade em rede (6. ed.) São Paulo: Paz e Terra.

Coskun, V., Ozdenizci, B., \& Ok, K. (2013). A survey on Near Field Communication (NFC) Technology. Wireless
Personal Communications, 71(3), 2259-2294. http://dx.doi. org/10.1007/s11277-012-0935-5.

Costa, F., Silva, H., Cavalcante, J., \& Araujo, A. (2010). Modelo de simulação computacional para avaliar a implantação da tecnologia de identificação por rádio frequência (RFID) em uma indústria de telefones celulares. In Anais do XXX Encontro Nacional de Engenharia de Produção (ENEGEP). Rio de Janeiro: ABEPRO.

Duroc, Y., \& Kaddour, D. (2012). RFID potential impacts and future evolution for Green projects. Energy Procedia, 18, 91-98. http://dx.doi.org/10.1016/j.egypro.2012.05.021.

Dutta, S. (2011). The Global Innovation Index 2011: accelerating growth and development. Fontainebleau: INSEAD.

Freiberger, A., \& Bezerra, M. B. P. (2010). RFID e seus impactos na logística. Logística Descomplicada.

Gil, A. C. (2009). Como elaborar projetos de pesquisa (4. ed.). São Paulo: Atlas.

Guisto, D., Iera, A., Morabito, G., \& Atzori, L. (2010). The internet of things. Springer.

Harrison, C., \& Donnelly, I. A. (2011). A theory of smart cities. In Proceedings of the 55th Annual Meeting of the International Society for the Systems Science (p. 1-15).

Meyer, G. G., Roest, G. B., \& Szirbik, N. B. (2010). Intelligent products for monitoring and control of road-based logistics. In Proceedings of the 4th IEEE International Conference on Management and Service Science (MASS'10). Wuhan-China.

Moovit. (2016). Sobre o Moovit. Recuperado em 22 de março de 2016, de http://www.moovitapp.com/pt-br/

Navarro, C.; Grillo, A.; \& Lima, R. (2008). Análise e proposição de melhorias no processo logístico em uma multinacional de tecnologia de informação e automação. In Anais do XXVIII Encontro Nacional de Engenharia de Produção. Rio de Janeiro: ABEPRO.

NFC Brasil. (2013). Projeto de São Paulo testa NFC em ônibus da Baixada Santista. Recuperado em 12 de abril de 2016, de http://nfcbrasil.wordpress.com/2013/01/18/ projeto-de-sao-paulo-testa-nfc-em-onibus-da-baixada-santista NFC Fórum. (2011). NFC in Public Transport. Recuperado em 11 de abril de 2016, de http://www.nfc-forum.org/ resources/white_papers/NFC_in_Public_Transport.pdf

Ok, K., Aydin, M., Coskun, V., \& Ozdenizci, B. (2011). Exploring underlying values of NFC applications. In Proceedings of the 
3rd International Conference on Information and Financial Engineering. Shanghai.

Ozdenizci, B., Aydin, M. N., Coskun, V., \& Ok, K.(2010). NFC research framework: a literature review and future research directions. Proceedings of the 14th IBIMA International Business Information Management Conference (p. 26722685). Istanbul.

RFID Journal. (2013a). Sistema Sem Parar/Via Fácil para pagamento de pedágios terá upgrade em 2013. Recuperado em 12 de abril de 2016, de http://brasil.rfidjournal.com/ noticias/vision?9320/1

RFID Journal. (2013b). Projeto com RFID pode tornar o pedágio mais justo nas estradas de São Paulo. Recuperado em 12 de abril de 2016, de http://brasil.rfidjournal.com/ noticias/vision?9662/2

Rosa, L. A. (2006). Aplicação do RFID na cadeia logística (monografia). Universidade de São Paulo, Escola Politécnica, São Paulo.

Soares, R. S., Comucci, T. L., Santos, T. C., Almeida, T. B., \& Leite, P. R. (2008). O Impacto da Tecnologia de Etiqueta Inteligente (RFID) na performance de cadeias de suprimentos: um estudo no Brasil. Revista Jovens Pesquisadores, 9(2), 101-118.
SPTrans. (2016). Olho Vivo: sistema de monitoramento do transporte. Recuperado em 22 de março de 2016, de http://olhovivo.sptrans.com.br/

Sterling, B. (2005). Shaping things mediawork pamphlets. Cambridge: The MIT Press.

Sun, C. (2012). Application of RFID technology for logistics on internet of things. In Proceedings of the AASRI Conference on Computational Intelligence and Bioinformatics.

Toppeta, D. (2010). The smart city vision: how innovation and ICT can build smart, "livable", sustainable cities. Milão: The Innovation Knowledge Foundation.

U.S. Departament of Transportation - US DOT. (2009). Research and innovative technology administration. Washington.

Waze. (2016). Waze é construir e se beneficiar de um bem comum nas ruas. Recuperado em 21 de março de 2016, de https://www.waze.com/pt-BR

WBus. (2016). Sobre o WBus. Recuperado em 22 de março de 2016, de http://www.wbus.net.br/

Recebido: Out. 14, 2015

Aprovado: Jul. 22, 2016 